\title{
"Positive to Negative" Dix-Hallpike test and Benign Paroxysmal Positional Vertigo recurrence in elderly undergoing Canalith Repositioning Maneuver and Vestibular Rehabilitation
}

\author{
Karyna M. O. B. de Figueiredo Ribeiro ${ }^{1}$ Lidiane Maria de Brito Macedo Ferreira ${ }^{2}$ \\ Raysa Vanessa de Medeiros Freitas ${ }^{1}$ Camila Nicácio da Silva ${ }^{1}$ Nandini Deshpande ${ }^{3}$ \\ Ricardo Oliveira Guerra ${ }^{1}$
}

1 Department of Physiotherapy, Universidade Federal do Rio Grande do
Norte, Natal, Rio Grande do Norte, Brazil
2 Post-Graduation Program in Public Health, Universidade Federal do
Rio Grande do Norte, Natal, Brazil
3 Faculty of Health Sciences, School of Rehabilitation Therapy, Queen's
University, Kingston, Canada

Int Arch Otorhinolaryngol 2016;20:344-352.

\begin{abstract}
Address for correspondence Karyna M. O. B. de Figueiredo Ribeiro, PhD, Department of Physiotherapy, Universidade Federal do Rio Grande do Norte, Senador Salgado Filho Avenue, n 3000, Campus Natal, Rio Grande do Norte 59078-970, Brazil

(e-mail: karynamy@hotmail.com).
\end{abstract}

\begin{abstract}
Introduction Benign Paroxysmal Positional Vertigo is the most common cause of dizziness in elderly people. Recent studies have shown that the elderly present higher Benign Paroxysmal Positional Vertigo recurrence and that vertiginous symptomatology remission varies according to comorbidities and the therapeutic techniques applied. Objective To assess the short-term effectiveness of Vestibular Rehabilitation in addition to Canalith Repositioning Maneuver on positive to negative Dix-Hallpike test, on recurrence and number of maneuvers to achieve a negative test in elderly patients with chronic Benign Paroxysmal Positional Vertigo.

Methods In this randomized controlled trial, 7 older adults (median age: 69 years, range 65-78) underwent Canalith Repositioning Maneuver and Vestibular Rehabilitation for thirteen weeks. Seven older adults (median age: 73 years, range 65-76) in the control group received only Canalith Repositioning Maneuver. The participants were assessed at baseline (T0), one (T1), five (T5), nine (T9), and thirteen weeks (T13). We assessed the differences between the groups by Mann-Whitney and Fisher exact tests, and used the Friedman and Wilcoxon tests to determine the intragroup differences. Results No significant differences were found between groups for the positive to negative Dix-Hallpike test, recurrence, and number of maneuvers to achieve a negative test. The number of maneuvers to achieve negative Dix-Hallpike test was lower in intragroup comparisons in the experimental group.

Keywords

- elderly

- vestibular diseases

- rehabilitation

Conclusion The findings suggest that additional Vestibular Rehabilitation did not influence the positive to negative Dix-Hallpike test, recurrence, or number of maneuvers to achieve a negative test in elderly patients with chronic Benign Paroxysmal Positional Vertigo.
\end{abstract}

received

September 15, 2015

accepted

November 15, 2015

published online

February 16, 2016
DOI http://dx.doi.org/

$10.1055 / \mathrm{s}-0036-1572528$. ISSN 1809-9777.
Copyright $(2016$ by Thieme Publicações License terms

Ltda, Rio de Janeiro, Brazil
(ब) $\Theta \circledast$ 


\section{Introduction}

Dizziness is a common symptom, especially in the elderly. It is estimated that $30 \%$ of people over the age of 65 complain of dizziness. The rate of annual consultations due to dizziness in primary care increases from $8 \%$ for individuals aged 65 years or older to $18 \%$ among those aged 85 or older. ${ }^{1,2}$ In elderly patients, this symptom is considered a geriatric multifactorial syndrome, caused by the physiological processes of aging and/or pathological processes. ${ }^{3-6}$

Benign Paroxysmal Positional Vertigo (BPPV) is the most common cause of dizziness in the adult population; 30\% of seniors over the age of 70 will experience at least one episode of BPPV throughout their life. ${ }^{7,8} \mathrm{BPPV}$ is classified according to its nature, in primary or idiopathic (considered the most common form of the disease), ${ }^{9}$ or secondary. The main causes for secondary BPPV are: traumatic brain injury, post-operative ear surgery, vertebrobasilar insufficiency, vestibular neuronitis, Ménière's disease or metabolic disorders. ${ }^{10}$ BPPV recurrences are associated with known risk factors such as diabetes mellitus, hypertension, osteoporosis and osteoarthritis. ${ }^{11}$

Posterior canal BPPV is considered the most common type of the disease, and is diagnosed by Dix-Hallpike test. ${ }^{12}$ The treatment can be based on non-pharmacological measures, highlighted by the Canalith Repositioning Maneuver (CRM) or modified Epley maneuver, a therapeutic recommendation with strongly proven evidence, and Vestibular Rehabilitation (VR) as a secondary option. ${ }^{13,14}$

The objectives of the study were to analyze the effectiveness of VR associated with the CRM compared with CRM in a "positive to negative" Dix-Hallpike test, BPPV recurrence, and the number of maneuvers to achieve a negative test in elderly patients with chronic BPPV. In addition, we aimed to trace the clinical profile of elderly with chronic BPPV and descriptively evaluate the associations of this (profile) with risk factors described in the literature.

\section{Method}

This is a randomized, single-blind, two-arm controlled trial that followed the recommendations established by the CONSORT (Consolidated Standards of Reporting Trials), 2010, ${ }^{15}$ recorded in the REBEC (Registro Brasileiro de Ensaios Clínicos) under code: RBR-7jkbyg. It was approved by the local Ethics Committee in Research, protocol 543/11, in accordance to resolution 196/96.

Seniors with chronic BPPV (minimum of 6 months) were referred from the otoneurology outpatient clinic of Hospital Universitário Onofre Lopes, Natal, RN, Brazil and from other public and private services of medical specializations that treat patients with complaints of dizziness. The study included individuals aged 65 years and older; having a positive Dix-Hallpike test with vertigo accompanied or not with positional nystagmus (objective or subjective BPPV). Exclusion criteria were: presence of cervical neurological symptoms; limited cervical amplitude of movement and instability for the implementation of both the diagnostic
Dix-Hallpike test and CRM; systemic diseases (such as hypertension, diabetes mellitus, and thyroid dysfunction) with no medication control; motor, hearing, or visual restrictions that prevent proper conduction of body balance evaluation and VR protocol; subjects not being able to understand and respond to simple verbal commands; having undergone previous treatment with CRM; and that had been performing some physical activity such as muscle strengthening, pilates, yoga, or high intensity aerobic exercises. Seniors who had some serious health complications which prevented the continuation of treatment or who did not want to continue the instituted therapy were also excluded from the study. Participants were informed about the procedures and objectives of the study and those who agreed to participate were asked to sign the Informed Consent Form.

After screening for the definition of the inclusion and exclusion criteria, the randomization process was performed in blocks with a sequence generated by a computer program (www.randomization.com). Patients were then randomized into two groups: 1 - Control Group (CG) - CRM; and 2 - Experimental group (EG) - CRM and additional VR. An independent researcher not involved with clinical trial did the process of randomization and allocation. Blinded investigators, different from those who performed the VR protocol performed the evaluations. Participants were assessed at baseline (T0) and at one (T1), five (T5), nine (T9), and thirteen (T13) weeks.

A multidimensional clinical questionnaire was used to collect sociodemographic (age and sex) and anthropometric data (body mass index in $\mathrm{kg} / \mathrm{m}^{2}$ ); anamnesis for BPPV with questions about nature (primary or secondary) and type of dizziness ${ }^{16}$ (vertigo, floating sensation, disequilibrium or presyncope), dizziness time onset, Dix-Hallpike test (positive or negative), presence of nystagmus, affected side (right, left, or bilateral), number of maneuvers, associated symptoms such as tinnitus; related health data, including comorbidities and medication use. We evaluated BPPV recurrence taking into account the "positive to negative" Dix-Hallpike test with subsequent positive results in the 13-week assessment period.

The classification for drug use was performed using the Anatomical Therapeutic Chemical Classification System (ATC), an alphanumeric coding system developed by the World Health Organization (WHO) for the classification of drugs and other medical products. The classification was in accordance with the second level. ${ }^{17}$ Polypharmacy occurrence was defined by concomitant use of five or more medications. $^{18}$

Regarding the associated diseases, the classification system we used was the International Classification of Diseases (ICD - 10). This is the international standard diagnostic classification for epidemiological and health administrative purposes, including analysis of the general situation of population groups and monitoring of incidence and prevalence of diseases and other health problems. ${ }^{19}$

Seniors were submitted to the Dix-Hallpike test ${ }^{20,21}$ with the aid of a Computerized Videonystagmoscopy Infrared System (SVNC, Contronic, Brazil), introduced to the least symptomatic side, according to information obtained from 
"Positive to Negative" Dix-Hallpike test and Benign Paroxysmal Positional Vertigo recurrence in elderly undergoing

each patient. If patients failed to report which position was responsible for triggering vertigo, the test started on the right side. Hence, the side that should be treated by CRM was identified. We observed eye movement for 1 minute in search of nystagmus and the patient was asked about the presence of vertigo. The same procedure was repeated with the head rotated to the other side. To confirm the involvement of the posterior semicircular canal, nystagmus should last less than one minute and be of the superior torsional type for canalithiasis cases; and one minute or slightly more for cupulolithiasis. ${ }^{22-24}$ Cases where dizziness during testing was present with or without positional nystagmus association were accepted. We conducted the Epley maneuver in accordance with the description of Epley (1992), but none of the patients received premedication prior to treatment or applied mastoid bone vibration. ${ }^{25,26} \mathrm{CRM}$ was performed up to 3 times in the same session if signs and symptoms persisted. ${ }^{22,23}$ Patients were warned that treatment could cause dizziness and nausea prior to each maneuver and they were asked to relax their neck muscles to prevent injury to the area. Orientations were given after completing the maneuvers. Next, CRM seniors were instructed to avoid rotation and extreme flexion-extension head movements, to avoid lying down on the affected side for 48 hours, and to elevate the head of the bed while sleeping for 24 hours. ${ }^{26}$ The medical assistant consented to suspend the anti-vertigo and anti-emetic preparations a week before the start of treatment.

After one week (T1), the seniors performed the same initial evaluation protocol, and those who had positive Dix-Hallpike test underwent CRM again, in addition to VR protocol for seniors belonging to the EG. The same happened at five (T5), nine (T9) and thirteen (T13) weeks after the initial assessment, totaling 5 assessments. The subjects of the EG performed customized VR based on other publications in the field, ${ }^{27,28}$ twice a week for a period of twelve weeks, supervised by trained physiotherapists. The sessions lasted an average of 50 minutes. The main deficits and functional limitations were identified in the initial assessment and the prescribed exercises were addressed to specific problems of the elderly. ${ }^{29}$ In customized exercise programs, the therapist regularly assesses the patient's progress and promotes feedback to the patient about the proper way to carry out the exercises. It is believed that this type of exercise increases patient adherence to treatment. ${ }^{30}$

VR included adaptation exercises (VORx1) and vestibular habituation (head and trunk repeated movements), static and dynamic balance training, and lower limb muscle strengthening exercises. The exercise program aimed to stimulate the vestibular system and promote sensory recalibration. During VR, therapists supervised exercises to ensure proper head and body movement for enhanced results (according to the habituation precepts) and monitored wrong posture, promoting greater patient safety. ${ }^{27,28}$ For each prescribed exercise, a universal series containing 10 modifiers and progression patterns was followed to achieve the most challenging exercises. For more information, please see the detailed description previously published. ${ }^{27}$ Participants were instructed to report all complaints (for example dizziness, nausea, or pain) during or after the exercises, which justified modifying the exercises, and to not do any other physical activity during the study. They were also encouraged to perform the exercises described in a booklet at home once a day.

\section{Statistical Analysis}

The normality of the data was analyzed using the ShapiroWilk test. Fisher's exact test was used to analyze the differences between groups at baseline regarding the distribution of sex, physical exercises performance and the presence of nystagmus, while the Mann-Whitney test was used to analyze differences between groups according to age, number of comorbidities, medication and dizziness time onset.

We analyzed the differences between groups regarding numerical results obtained at baseline (T0), one (T1), five (T5), nine (T9), and thirteen (T13) weeks using the Mann-Whitney test. Fisher's exact test was also used for dichotomous variables (positive or negative Dix-Hallpike and recurrence). We used the non-parametric Friedman test to elucidate the differences in repeated intragroup measurements over time. We used the Wilcoxon test to compare intragroup improvement. We performed all statistical tests using version 20.0 of the Statistical Package for Social Sciences (SPSS) with statistical significance set at $p<0.05$.

\section{Result}

Fourteen subjects were randomly allocated to one of the two groups. In the experimental group, 6 women $(6 / 7,85.7 \%)$ and 1 man $(1 / 7,14.3 \%)$ with a median age of 69 years (65-78) underwent CRM whenever necessary and VR for thirteen weeks whereas, five women $(5 / 7,71.4 \%)$ and 2 men $(2 / 7,28.6 \%)$ with a median age of 73 years (65-76) received only CRM in the control group. The research flowchart is shown in - Fig. 1.

There were no statistical differences between groups at baseline for BPPV sociodemographic and clinical characteristics $(p<0.05)$. Body Mass Index $(p=0.720)$, dizziness time onset ( $p=0.169)$, presence of tinnitus ( $p=1.000)$, presence of nystagmus ( $p=1.000$ ), number of medications used by the elderly ( $p=0.386$ ), as well as the number of comorbidities ( $p=1.000)$ were also statistically similar for both groups at baseline. The median for the number of comorbidities in the experimental group was $4(3-6)$ and $4(2-6)$ in the control group. The median number of drugs used was $3(1-6)$ in the experimental group and 3 (3-7) in the control group. Other clinical characteristics of seniors participating in the study are presented in - Table 1.

In assessing the "positive to negative" Dix-Hallpike test, it was observed that all seniors in the EG obtained negative results after 13 weeks. In contrast, the CG showed treatment failure in $2 / 7(28.6 \%)$ patients. Though, there was no difference $(p>0.05)$ between both groups throughout the testing period (-Fig. 2).

Regarding the number of maneuvers performed in each session, we found no differences between the groups at baseline (T0), first (T1), fifth (T5), ninth (T9), and thirteenth (T13) week of assessment $(p>0.05)$. Only in the EG it is possible to observe significant improvement gradient, with a 


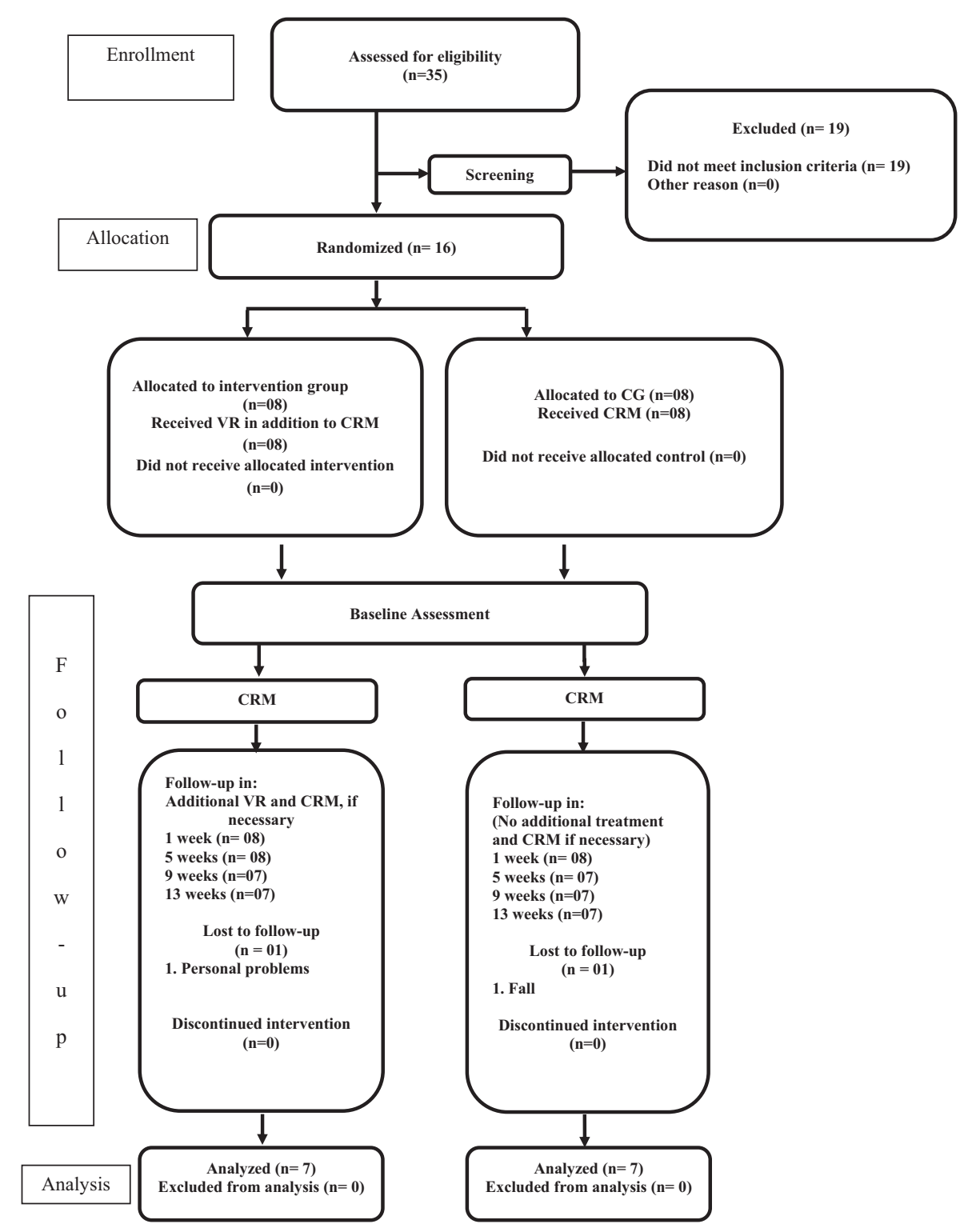

Fig. 1 Flowchart of the study. CRM, Canalith Repositioning Maneuvers; VR, Vestibular Rehabilitation.

progressive decrease in the number of maneuvers necessary for the treatment ( - Table 2 ).

Two patients $(2 / 7,28.3 \%)$ in the control group and $1(1 / 7$, $14.7 \%$ in the experimental group had recurrence of BPPV in the 13-week period. However, this fact did not favor the statistically significant difference between groups $(p=1.000)$. Among the seniors who had recurrence, Senior 1 had bilateral BPPV, hypertension as a risk factor for the disease, and used 6 daily medications. Senior 6 had diabetes as a risk factor and used 3 daily medications. Senior 8 had secondary BPPV and remained feeling dizziness (floating sensation) after 13 weeks. There were no adverse events or complications of treatment, such as posterior canal BPPV being converted to horizontal canal BPPV.

\section{Discussion}

Vestibular dysfunction is a major cause of dizziness in the elderly and represents $40-50 \%$ of the causes of dizziness in elderly patients referred to the otorhinolaryngologist. It is also described as an important differential diagnosis when the elderly falls without apparent cause. ${ }^{30}$ Chronically dizzy elderly have $53.3 \%$ of falls per year, ${ }^{31}$ and, although vertigo corresponds to $25 \%$ of falls, it is separately disclosed as the most common cause. ${ }^{7}$ In a recent cohort study, ${ }^{32}$ it was observed that BPPV elderly patients exhibited 1.14-fold higher risk to fracture compared with healthy elderly, when adjusted for age, sex, and comorbidities.

Non-pharmacological alternatives for the treatment of BPPV, including CRM, represent an important therapeutic opportunity for not presenting risks of adverse effects usually present in the elderly that use pharmacological products. The treatment may be associated with VR, which includes vestibular adaptation, habituation, and substitution exercises, associated with a set of procedures linked to changes in habits and postural improvement, ${ }^{21,30,33}$ despite the lack of substantial scientific evidence that VR enhances the effect of CRM or provides any benefit whatsoever. ${ }^{13,14}$ 
"Positive to Negative" Dix-Hallpike test and Benign Paroxysmal Positional Vertigo recurrence in elderly undergoing

\begin{tabular}{|c|c|c|c|c|c|c|c|c|}
\hline$\sum_{\bar{z}}^{\circ}$ & 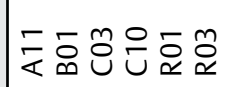 & 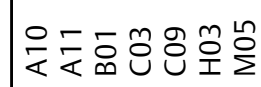 & 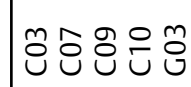 & ํㅜㅇํํ & 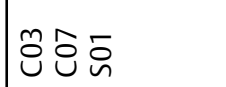 & 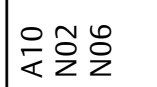 & $\overbrace{0}^{\infty} \frac{n}{2} \bar{z}$ & $\sum \bar{n}$ \\
\hline 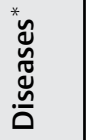 & 을 呈䍃 & 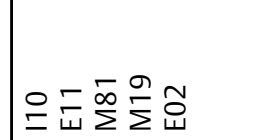 & 을 是点 & 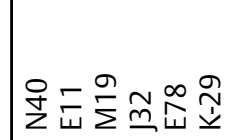 & 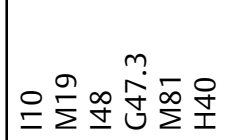 & 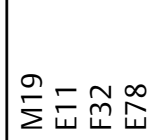 & $O \stackrel{\infty}{=}$ & 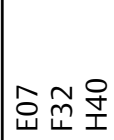 \\
\hline 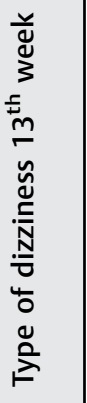 & 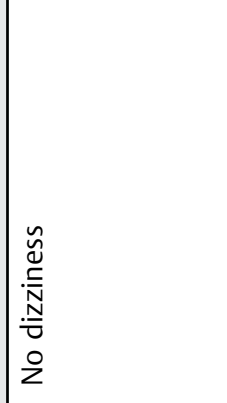 & 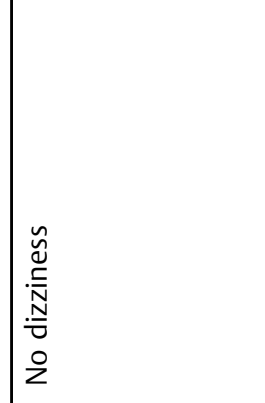 & 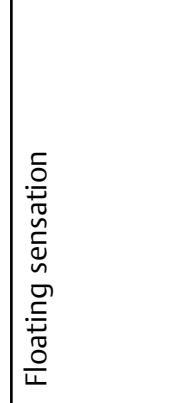 & 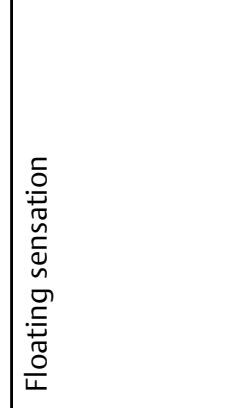 & 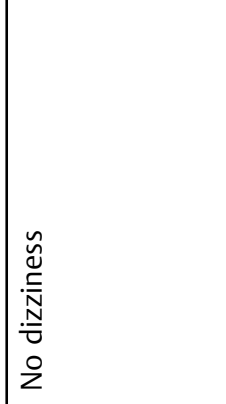 & 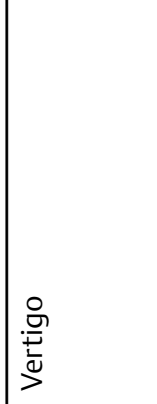 & 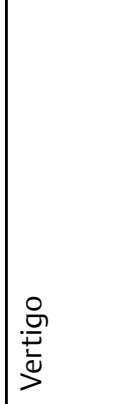 & 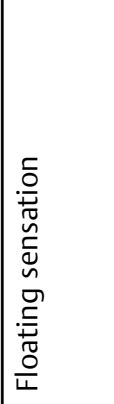 \\
\hline 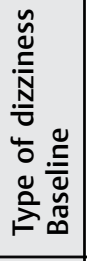 & 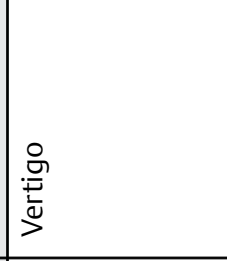 & 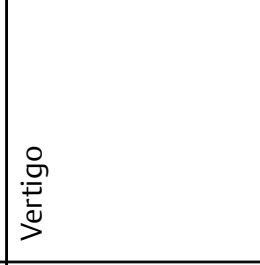 & 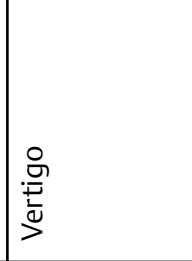 & 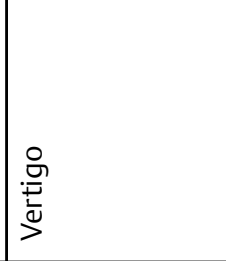 & 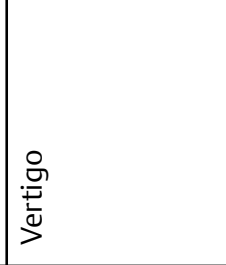 & 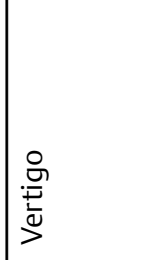 & 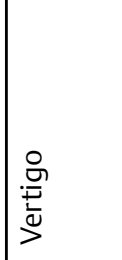 & 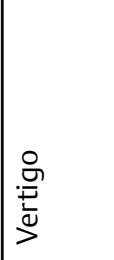 \\
\hline 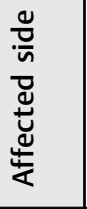 & 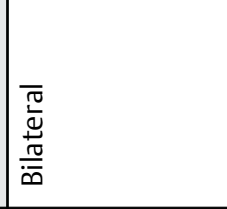 & 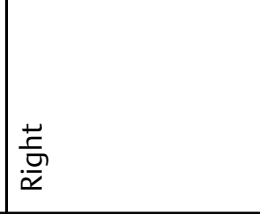 & \begin{tabular}{|l} 
䓂 \\
$\frac{a}{\alpha}$
\end{tabular} & 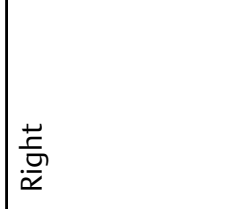 & 过 & \begin{tabular}{|l} 
䓂 \\
$\frac{G}{\alpha}$
\end{tabular} & $\mid \begin{array}{l}\text { 蒙 } \\
\frac{a}{\alpha}\end{array}$ & 蒙 \\
\hline 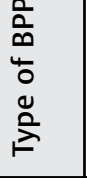 & 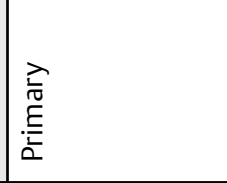 & 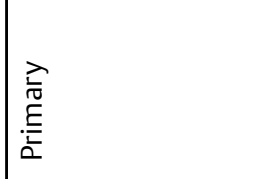 & 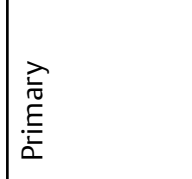 & \begin{tabular}{|l} 
离 \\
畜 \\
|
\end{tabular} & 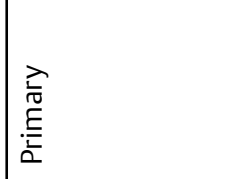 & 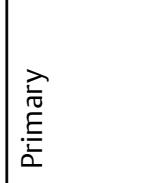 & 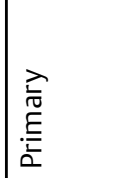 & 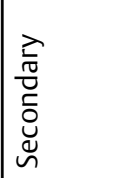 \\
\hline 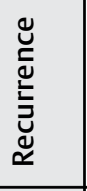 & $\stackrel{\check{\nu}}{\nu}$ & $\frac{9}{2}$ & 2 & $\frac{9}{2}$ & $\frac{9}{2}$ & $\stackrel{\check{\nu}}{\nu}$ & $\frac{9}{2}$ & $\stackrel{\check{\nu}}{-}$ \\
\hline 言 & U & U & U & U & u & u & u & u \\
\hline ๖ & 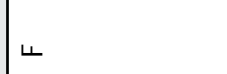 & L & $\Sigma$ & $\Sigma$ & L & L & | & 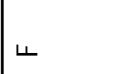 \\
\hline 娄 & 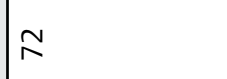 & it & m & 2 & it & $\mathbb{N}$ & 茴 & |ڤి \\
\hline & 吾 & 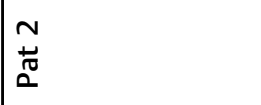 & 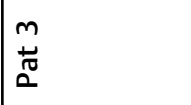 & 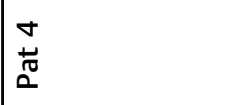 & $\underset{\substack{n \\
\Delta \\
c}}{\substack{n \\
c}}$ & $\begin{array}{l}0 \\
0 \\
0 \\
0\end{array}$ & 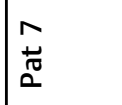 & 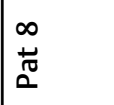 \\
\hline
\end{tabular}




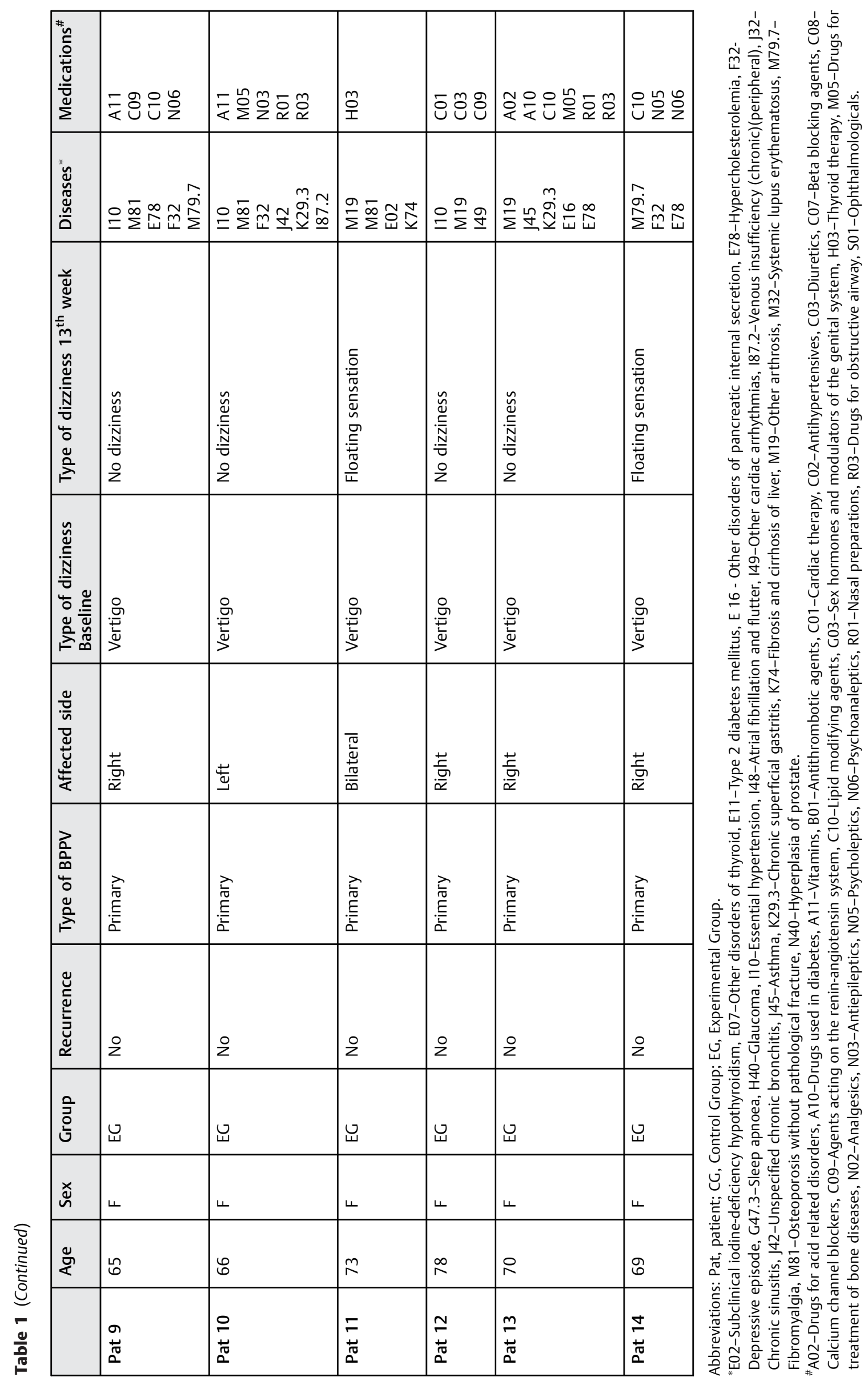


"Positive to Negative" Dix-Hallpike test and Benign Paroxysmal Positional Vertigo recurrence in elderly undergoing

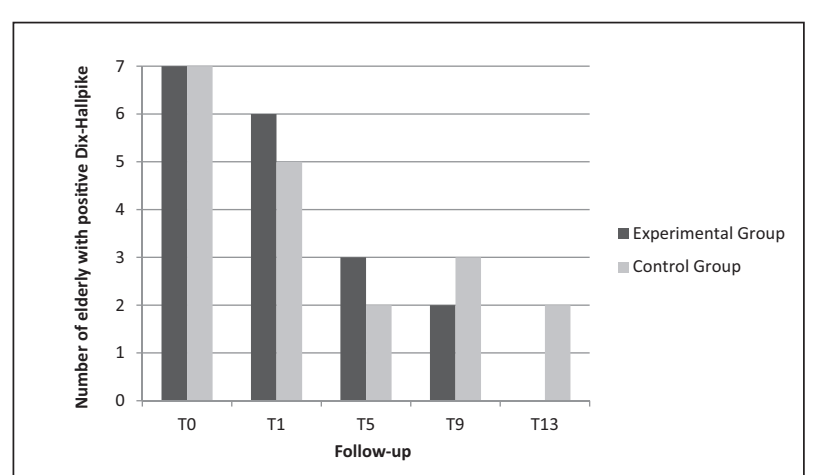

Fig. 2 Descriptive analysis of the "positive to negative" Dix-Hallpike test at baseline (T0), first (T1), fifth (T5), ninth (T9) and thirteenth week (T13) of assessment.

Our findings revealed that negative Dix-Hallpike test results or number of maneuvers to achieve it over the 13 weeks were not significantly different regardless of patients having performed additional VR therapy. However, it is important to mention that all patients in the EG obtained negative test results with consequent remission of vertigo after treatment with additional VR, unlike in the control group, which was unsuccessful with two seniors. It might be suggested that these findings porated into the VR program. Previous studies ${ }^{23,34}$ that performed vestibular habituation exercises in patients who had reported good results with this combination therapy.

BPPV recurrence is set at 1.7 -fold higher risk in elderly patients with BPPV than in younger individuals suffering with the same disease. ${ }^{35}$ In this study, the prevalence of symptoms recurrence over the 13-week follow-up was $21.4 \%$, corroborating with Dorigueto et al. ${ }^{34}$ In the literature, however, the recurrence rate variability shows a result of $26 \%{ }^{34}$ to $50 \%, 36,37$ which can be explained by the difference in time and patient follow-up mode. Choi et $\mathrm{al}^{38}$ observed that patients with BPPV presenting recurrence needed longer follow-up and CRM treatment periods when compared with groups that achieved rapid remission or symptom persistence.

Of the three seniors who presented recurrence in this study, one had hypertension and one had associated osteoarthritis and diabetes. A recent multicenter study ${ }^{11}$ demonstrated that the association between two or more comorbidities such as hypertension, diabetes, and osteoarthritis are able to significantly influence the number of BPPV recurrences and that osteoporosis is associated with a higher risk for such condition. Since this was a study conducted with elderly who have more comorbidities than the general population, ${ }^{39}$ the likelihood of recurrence is higher because these associated diseases, recognized as BPPV triggers, are more present in such individuals.

BPPV can recur when secondary to some neurological or otological event. In this study, the only senior who had secondary BPPV (which was due to prolonged bed rest from being in a coma, also considered a triggering event to $\mathrm{BPPV})^{40}$ had recurrence. Cases of recurrence and persistence of BPPV mostly result from the idiopathic form of the disease. occurred due to the habitual performance of exercises incorpersistent BPPV symptoms after treatment through CRM

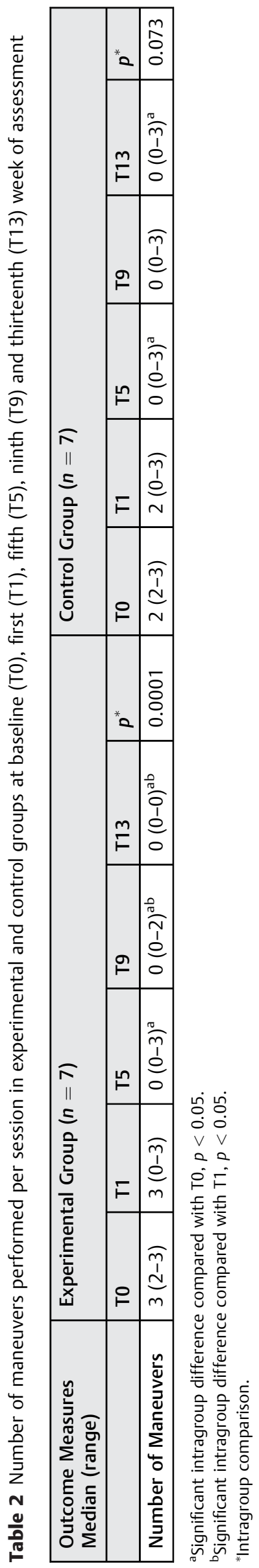

International Archives of Otorhinolaryngology Vol. 20 No. 4/2016 
Additionally, individuals suffering from secondary BPPV are more likely to present vertigo symptoms for a longer time, and even after dizziness remission, may have disease recurrence. ${ }^{38,41}$

It was found that more than half of the elderly were suffering from hypertension, hypercholesterolemia, and arthritis, and that most also had osteoporosis and diabetes. Patients with BPPV have more vascular risk factors when compared with patients suffering from other vestibular diseases. ${ }^{42,43}$ Furthermore, elderly patients with BPPV have 1.3fold higher risk, adjusted by age and sex, to develop acute ischemic stroke than seniors who do not have the disease. ${ }^{44}$ Cardiovascular, metabolic, and osteoarticular diseases, and osteoporosis are risk factors for the onset of BPPV, and may also be related to its genesis., ${ }^{7,45-47}$

A considerable number of comorbidities per elderly was found. This may explain the low resolution of the symptomatology, since most of the surveyed elderly suffer from comorbidities that directly interfere with dizziness symptoms. The greater the number of associated diseases, the greater the risk for dizziness, especially if that number is greater than three. ${ }^{48,49}$ The high number of associated diseases may be related to the fact that some seniors improved the vertigo symptom related to BPPV, but remain feeling another type of dizziness. This occurred in five patients in this study (35.7\%). Those participants remained with floating sensation dizziness even after a negative Dix-Hallpike test. The low number of subjects involved in the study can be considered as a potential limitation on the extrapolation of results found. However, the form of selection, and type (randomized controlled clinical trial) are considered strengths of the study. The findings of the intervention and its clinical implications must be wisely considered for making therapeutic decisions in elderly patients with BPPV. Despite the fact that we did not find differences between groups, VR should be considered as an important adjuvant therapeutic tool, since the consequences arising out of BPPV such as falls and fractures in the elderly can be potentially avoided through CRM associated with postural balance training exercises. ${ }^{32}$ We suggest further studies to evaluate the effectiveness of VR with larger sample sizes and longer-term follow-up, as vestibular functions may be influenced by confounding factors, which may cause a change in the health status of the elderly throughout treatment.

\section{Conclusion}

In the present study, elderly patients with BPPV were mostly women and had a considerable number of comorbidities. The findings suggest that "positive to negative" Dix-Hallpike test, BPPV recurrence, and the number of maneuvers to achieve negative results were not influenced by additional VR to CRM in the elderly with chronic BPPV.

Conflicts of Interest

The authors declare no conflicts of interest.

\section{References}

1 Maarsingh OR, Dros J, Schellevis FG, van Weert HC, Bindels PJ, Horst HE. Dizziness reported by elderly patients in family practice: prevalence, incidence, and clinical characteristics. BMC Fam Pract 2010;11:2

2 Sloane PD, Coeytaux RR, Beck RS, Dallara J. Dizziness: state of the science. Ann Intern Med 2001;134(9 Pt 2):823-832

3 Horak FB. Postural orientation and equilibrium: what do we need to know about neural control of balance to prevent falls? Age Ageing 2006;35(2, Suppl 2):ii7-ii11

4 Jönsson R, Sixt E, Landahl S, Rosenhall U. Prevalence of dizziness and vertigo in an urban elderly population. J Vestib Res 2004; 14(1):47-52

5 Kutz JW Jr. The dizzy patient. Med Clin North Am 2010;94(5): 989-1002

6 Tinetti ME, Williams CS, Gill TM. Dizziness among older adults: a possible geriatric syndrome. Ann Intern Med 2000;132(5): 337-344

7 Ganança FF, Gazzola JM, Ganança CF, Caovilla HH, Ganança MM, Cruz OL. Elderly falls associated with benign paroxysmal positional vertigo. Braz J Otorhinolaryngol 2010;76(1):113-120

8 Cho EI, White JA. Positional vertigo: as occurs across all age groups. Otolaryngol Clin North Am 2011;44(2):347-360, viii

9 Moreno NS, André AP. Number of maneuvers need to get a negative Dix-Hallpike test. Braz J Otorhinolaryngol 2009;75(5): 650-653

10 Caldas MA, Ganança CF, Ganança FF, Ganança MM, Caovilla HH. Clinical features of benign paroxysmal positional vertigo. Braz J Otorhinolaryngol 2009;75(4):502-506

11 De Stefano A, Dispenza F, Suarez H, et al. A multicenter observational study on the role of comorbidities in the recurrent episodes of benign paroxysmal positional vertigo. Auris Nasus Larynx 2014; 41(1):31-36

12 Kerber KA, Burke JF, Skolarus LE, et al. Use of BPPV processes in emergency department dizziness presentations: a population-based study. Otolaryngol Head Neck Surg 2013;148(3): 425-430

13 Bhattacharyya N, Baugh RF, Orvidas L, et al; American Academy of Otolaryngology-Head and Neck Surgery Foundation. Clinical practice guideline: benign paroxysmal positional vertigo. Otolaryngol Head Neck Surg 2008;139(5, Suppl 4):S47-S81

14 Fife TD, Iverson DJ, Lempert T, et al; Quality Standards Subcommittee, American Academy of Neurology. Practice parameter: therapies for benign paroxysmal positional vertigo (an evidence-based review): report of the Quality Standards Subcommittee of the American Academy of Neurology. Neurology 2008; 70(22):2067-2074

15 Schulz KF, Altman DG, Moher D. CONSORT 2010 statement: updated guidelines for reporting parallel group randomized trials. Ann Intern Med 2010;152(11):726-732

16 Drachman DA, Hart CW. An approach to the dizzy patient. Neurology 1972;22(4):323-334

17 WHO Collaborating Centre for Drug Statistics Methodology. (2012) Guidelines for ATC Classification and DDD Assignment 2013. 16th ed. WHO Collaborating Centre for Drug Statistics Methodology, Oslo.

18 Flores LM, Mengue SS. Uso de medicamentos por idosos em região do sul do Brasil. Rev Saude Publica 2005;39(6):924-929

19 Saúde. OMd.CID-10, tradução do Centro Colaborador da OMS para a Classificação de Doenças em Português. 2003. 9ª ed. São Paulo: EDUSP

20 Gold DR, Morris L, Kheradmand A, Schubert MC. Repositioning maneuvers for benign paroxysmal positional vertigo. Curr Treat Options Neurol 2014;16(8):307

21 Silva AL, Marinho MR, Gouveia FM, Silva JG, Ferreira AdeS, Cal R. Benign Paroxysmal Positional Vertigo: comparison of two recent 
international guidelines. Braz J Otorhinolaryngol 2011;77(2): 191-200

22 Helminski JO, Zee DS, Janssen I, Hain TC. Effectiveness of particle repositioning maneuvers in the treatment of benign paroxysmal positional vertigo: a systematic review. Phys Ther 2010;90(5): 663-678

23 Angeli SI, Hawley R, Gomez O. Systematic approach to benign paroxysmal positional vertigo in the elderly. Otolaryngol Head Neck Surg 2003;128(5):719-725

24 Herdman SJ, Tusa RJ. Physical Therapy Management of Benign Positional Vertigo. In: Herdman SJ. Vestibular Rehabilitation. 3rd ed. Philadelphia, PA: F. A. Davis Company; 2007:233-60

25 Epley JM. The canalith repositioning procedure: for treatment of benign paroxysmal positional vertigo. Otolaryngol Head Neck Surg 1992;107(3):399-404

26 Bruintjes TD, Companjen J, van der Zaag-Loonen HJ, van Benthem PP. A randomised sham-controlled trial to assess the long-term effect of the Epley manoeuvre for treatment of posterior canal benign paroxysmal positional vertigo. Clin Otolaryngol 2014;39(1):39-44

27 Alsalaheen BA, Whitney SL, Mucha A, Morris LO, Furman JM, Sparto PJ. Exercise prescription patterns in patients treated with vestibular rehabilitation after concussion. Physiother Res Int 2013; 18(2):100-108

28 Chang WC, Yang YR, Hsu LC, Chern CM, Wang RY. Balance improvement in patients with benign paroxysmal positional vertigo. Clin Rehabil 2008;22(4):338-347

29 Herdman SJ, Whitney SL. Interventions for the Patient with Vestibular Hypofunction. In: Herdman SJ. Vestibular Rehabilitation. 3rd ed. Philadelphia, P.A.: f.a. Davis Company; 2007: 300937Herdman SJ WS. Interventions for the patient with Vestibular Hypofunction. 2007:309-37

30 Alrwaily M, Whitney SL. Vestibular rehabilitation of older adults with dizziness. Otolaryngol Clin North Am 2011;44(2):473-496, x

31 Gazzola JM, Ganança FF, Aratani MC, Perracini MR, Ganança MM. Circumstances and consequences of falls in elderly people with vestibular disorder. Braz J Otorhinolaryngol 2006;72(3):388-392

32 Liao WL, Chang TP, Chen HJ, Kao CH. Benign paroxysmal positional vertigo is associated with an increased risk of fracture: a population-based cohort study. J Orthop Sports Phys Ther 2015;45(5): 406-412

33 Whitney SL, Sparto PJ. Principles of vestibular physical therapy rehabilitation. NeuroRehabilitation 2011;29(2):157-166

34 Dorigueto RS, Mazzetti KR, Gabilan YP, Ganança FF. Benign paroxysmal positional vertigo recurrence and persistence. Braz J Otorhinolaryngol 2009;75(4):565-572
35 Kao CL, Hsieh WL, Chern CM, Chen LK, Lin MH, Chan RC. Clinical features of benign paroxysmal positional vertigo (BPPV) in Taiwan: differences between young and senior age groups. Arch Gerontol Geriatr 2009; 49(2, Suppl 2):S50-S54

36 Baloh RW, Honrubia V, Jacobson K. Benign positional vertigo: clinical and oculographic features in 240 cases. Neurology 1987; 37(3):371-378

37 Brandt T, Daroff RB. Physical therapy for benign paroxysmal positional vertigo. Arch Otolaryngol 1980;106(8):484-485

38 Choi SJ, Lee JB, Lim HJ, et al. Clinical features of recurrent or persistent benign paroxysmal positional vertigo. Otolaryngol Head Neck Surg 2012;147(5):919-924

39 Batuecas-Caletrio A, Trinidad-Ruiz G, Zschaeck C, et al. Benign paroxysmal positional vertigo in the elderly. Gerontology 2013; 59(5):408-412

40 Charles C, Santina D, Minor LB, Carey JP In: Lee KJ. Princípios de Otorrinolaringologia: cirurgia de cabeça e pescoço. 9th ed. Porto Alegre, RS: AMGH editora; 2010:94-134

41 Tanimoto H, Doi K, Nishikawa T, Nibu K. Risk factors for recurrence of benign paroxysmal positional vertigo. J Otolaryngol Head Neck Surg 2008;37(6):832-835

42 De Reuck J. Vascular risk factors in patients with peripheral vestibular disorders. Acta Neurol Belg 2010;110(4):303-305

43 Wada M, Naganuma H, Tokumasu K, Hashimoto S, Ito A, Okamoto M. Arteriosclerotic changes as background factors in patients with peripheral vestibular disorders. Int Tinnitus J 2008;14(2):131-134

$44 \mathrm{Kao} \mathrm{CL}$, Cheng YY, Leu HB, et al. Increased risk of ischemic stroke in patients with benign paroxysmal positional vertigo: a 9-year follow-up nationwide population study in taiwan. Front Aging Neurosci 2014;6(2):108

45 Gazzola JM, Ganança FF, Aratani MC, Perracini MR, Ganança MM. Clinical evaluation of elderly people with chronic vestibular disorder. Braz J Otorhinolaryngol 2006;72(4):515-522

46 Bittar RS, Pedalini ME, Ramalho JO, Yoshimura R. Critical analysis of vestibular rehabilitation outcome according to dizziness etiology. Braz J Otorhinolaryngol 2007;73(6):760-764

47 Yu S, Liu F, Cheng Z, Wang Q. Association between osteoporosis and benign paroxysmal positional vertigo: a systematic review. BMC Neurol 2014;14:110

48 Gassmann KG, Rupprecht R. Dizziness in an older community dwelling population: a multifactorial syndrome. J Nutr Health Aging 2009;13(3):278-282

49 Gomez F, Curcio CL, Duque G. Dizziness as a geriatric condition among rural community-dwelling older adults. J Nutr Health Aging 2011;15(6):490-497 